\title{
Maternal Postpartum Depression and Infant Psychomotor Development: Descriptive Study of a Population Hospitalized in the Mother and Baby Unit
}

\author{
Le Treut $\mathbf{L}^{1 *}$, Poinso $\mathrm{F}^{1}$, Grandgeorge $\mathbf{P}^{1}$, Dugnat $\mathbf{M}^{1}$, Sparrow $\mathrm{J}^{\mathbf{2}}$ and Guivarch $\mathbf{J}^{1}$ \\ ${ }^{1}$ Department of Child Psychiatry, Sainte Marguerite Hospital, APHM, Aix-Marseille University, France \\ ${ }^{2}$ Brazelton Touchpoints Center, Division of Developmental Medicine Boston Children's Hospital/Harvard Medical School, USA
}

\begin{abstract}
Little evaluation has been done on the psychomotor development of babies in cases of maternal psychiatric pathology in the first year. The Mother and Baby Unit (MBU) is a psychiatric hospital healthcare system available to infants and their mothers during the postpartum period.
\end{abstract}

Aim: To describe the development of babies of mothers with severe postpartum depression treated in MBU.

Methods: Using the Brunet-Lézine scale, we studied six-month-old infants whose mothers were hospitalized.

Results: The study population consisted of 14 infants. We found no global psychomotor development delay. That aside, posture was the subscore area where we observed the most difficulties.

Conclusion: It is possible that the tonic dialogue between mother and infant is disrupted by maternal depression, both on an emotional level and through maternal psychomotor retardation. We would like to draw attention to the advantages of a more objective development metric than daily observations of babies' behavior.

Keywords: Maternal postpartum depression; Infant psychomotor development; Revised Brunet-Lézine scale; Mother and baby unit

\section{Introduction}

The Mother and Baby Unit (MBU) is a psychiatric hospital level three healthcare system available in France to infants and their mothers during the postpartum period. It is tasked with treating puerperal maternal disorders and relationship difficulties between mothers and infants [1-3]. They play a preventive and early intervention role in child development.

Indications for joint hospitalization include initial or recurring episodes of postpartum psychiatric disorders, acute or chronic maternal psychiatric pathologies, mother-baby relationship disorders, functional disorders and some infant development delays. The approach to treating mothers during mother-infant hospitalization is twofold: treatment of the psychiatric disorder, and evaluation and support of the developing mother-infant interactions [1]. For the infant, the institution ensures that physical and psychiatric care is safe and consistent [4]. This does not mean that the institution is replacing the mother, but rather that it defers to the mother when she is providing the necessary care, and steps in to compensate at times when maternal care is unavailable or the pathological process hinders mother-infant interaction. Relational challenges, even as early as the first year, have been correlated with the development of abnormal psychology in children, some cases of psychomotor retardation and difficulty forming attachments $[5,6]$.

\section{Method}

\section{plan and population}

We performed a cross-sectional descriptive study of the development of infants of mothers with depression, at the age of six months, using the revised Brunet-Lézine scale.

Inclusion criteria: Mother experiencing a first Severe Depressive Episode during the postnatal period. The diagnosis was made according to DSM-IV criteria by the MBU's two senior psychiatrists mother with an infant aged six months, give or take one month. Both parents agreement obtained for the anonymized use of data.

Exclusion criteria: Mothers with a different pathology (anxiety disorders, psychotic disorders, borderline disorders with no associated signs of depressive episodes) infant discharged before the age of six months or admitted after the age of six months

\section{Judgement criterion: The Brunet-lezine scale}

The revised Brunet-Lézine test is one of the only development scales for young children that has been calibrated on a French sample. It is similar to the Bayley test [7-9]. The Brunet-Lézine is the test most often used in France in the field of infant development evaluation. Four major development areas are studied: posture DQ, coordination DQ, sociability DQ, and language DQ. The Global Development Quotient (global DQ) is calculated from these sub-scores. The normal range is from 80 to 120 with a mean of 100 . The revised Brunet-Lézine scale provides an assessment at a time $\mathrm{T}$ in the child's development.

\section{Measurement}

A psychologist external to the treatment team administers the Brunet-Lézine scale tests in an attempt to provide a more objective point of view on the impact of relational challenges on the baby. We

*Corresponding author: Le Treut L, Department of Child Psychiatry, Sainte Marguerite Hospital, APHM, 270 Boulevard de Sainte Marguerite, 13009 Marseille, France, Tel: +33 4 91744767; Fax: 334 91746242; E-mail: Laure.LE-TREUT@ap-hm.fr.

Received June 15, 2017; Accepted July 06, 2017; Published July 10, 2017

Citation: Le Treut L, Poinso F, Grandgeorge P, Dugnat M, Sparrow J, et al. (2017) Maternal Postpartum Depression and Infant Psychomotor Development: Descriptive Study of a Population Hospitalized in the Mother and Baby Unit. J Ment Disord Treat 3: 138. doi:10.4172/2471-271X.1000138

Copyright: $\odot 2017$ Le Treut L, et al. This is an open-access article distributed under the terms of the Creative Commons Attribution License, which permits unrestricted use, distribution, and reproduction in any medium, provided the original author and source are credited. 
Citation: Le Treut L, Poinso F, Grandgeorge P, Dugnat M, Sparrow J, et al. (2017) Maternal Postpartum Depression and Infant Psychomotor Development: Descriptive Study of a Population Hospitalized in the Mother and Baby Unit. J Ment Disord Treat 3: 138. doi:10.4172/2471271X.1000138

Page 2 of 3

consider an infant to have a true delay only if his or her global DQ has a score of less than 70 .

\section{Statistical methods}

Statistical analysis is primarily descriptive. The qualitative variables are described by numbers and percentages of patients within each group. The quantitative variables (DQ and subscores) are reported with means, standard deviation and medians.

\section{Ethics committee}

The protocol has been submitted to the University of Aix-Marseilles Ethics Committee, which has granted its approval.

\section{Results}

\section{Description of the study population}

The study population consisted of 13 dyads and 14 infants (one dyad included twins) successively treated in the mother and baby unit in the Saint-Marguerite University Hospital Center in Marseille between 2014 and 2016.

The mothers' main diagnosis was severe postpartum depression. Two of the 13 mothers had borderline personality disorder; one had a depressive episode in the context of a bipolar disorder. There were 14 infants, ten boys and four girls. The mean age of the babies was six

\begin{tabular}{|c|c|c|c|}
\hline Variables & N & $\%$ \\
\hline \multirow{2}{*}{ Child's sex } & Child's age & 10 & 71.4 \\
\hline \multirow{2}{*}{ Mother's diagnosis } & Girl & 4 & 28.6 \\
\cline { 2 - 4 } & \multicolumn{1}{|c|}{$\begin{array}{c}\text { Postpartum depression } \\
\text { Postpartum depression + borderline } \\
\text { personality disorder }\end{array}$} & 2 & 10.4 \\
\cline { 2 - 4 } & $\begin{array}{l}\text { Postpartum depression + bipolar disorder } \\
\text { Other parameter }\end{array}$ & 1 & 7.7 \\
\hline Mother's age & Mean years and five months & \multicolumn{2}{|c|}{} \\
\hline Child's age & Six months and 13 days & \multicolumn{2}{|c|}{} \\
\hline
\end{tabular}

Table 1: Population characteristics. months and 13 days. The main characteristics of the population are laid out in Table 1.

Description of the children's development quotients (DQ): The mean global DQ score was 97.6, the median was 97 and the range was 108 to 88 . One child had a global DQ score of under 90. Regarding DQ subscores, the mean posture DQ score was 94 (median 96); the mean coordination DQ score was 98.8 (median 99.5); the mean language DQ score was 104.1 (median 101.5); and the mean sociability DQ score was 95.8 (median 98). The main development scale results are displayed in Figure 1.

\section{Discussion}

The main result of our study was the lack of developmental delay among the infants in our population. Only one child fell in the "average-low" zone with a global DQ score of 88. These results are consistent with observations of Piteo et al. who studied 18 months infant development whose mother suffered from postpartum depression (Piteo, Yelland, and Makrides, 2012). Although it was not pathological, the posture category was the index where we observed the most difficulties in the population of children studied. One child was actually in the "borderline" zone (score of 70-80). Three other children had results in the "average-low" zone (score of 80-90), accounting for $21.4 \%$ of the population. A total of four children, or $28.5 \%$, had low results on this index.

These children of depressive mothers did not, therefore, present any impaired development in the first months of life, but some of them seemed fragile in terms of the posture category.

It is possible that the tonic dialogue described by Wallon [10] and Ajurriaguerra [11] is disrupted by maternal depression, both on an emotional level and through maternal psychomotor retardation. Tonic dialogue is one of the ways to help the baby with tonic regulation by metabolizing body tension accumulated in reaction to sensory stimuli and giving it meaning. For Bullinger [12], tonic dialogue meets a vital communication need for the baby. We can theorize that postpartum

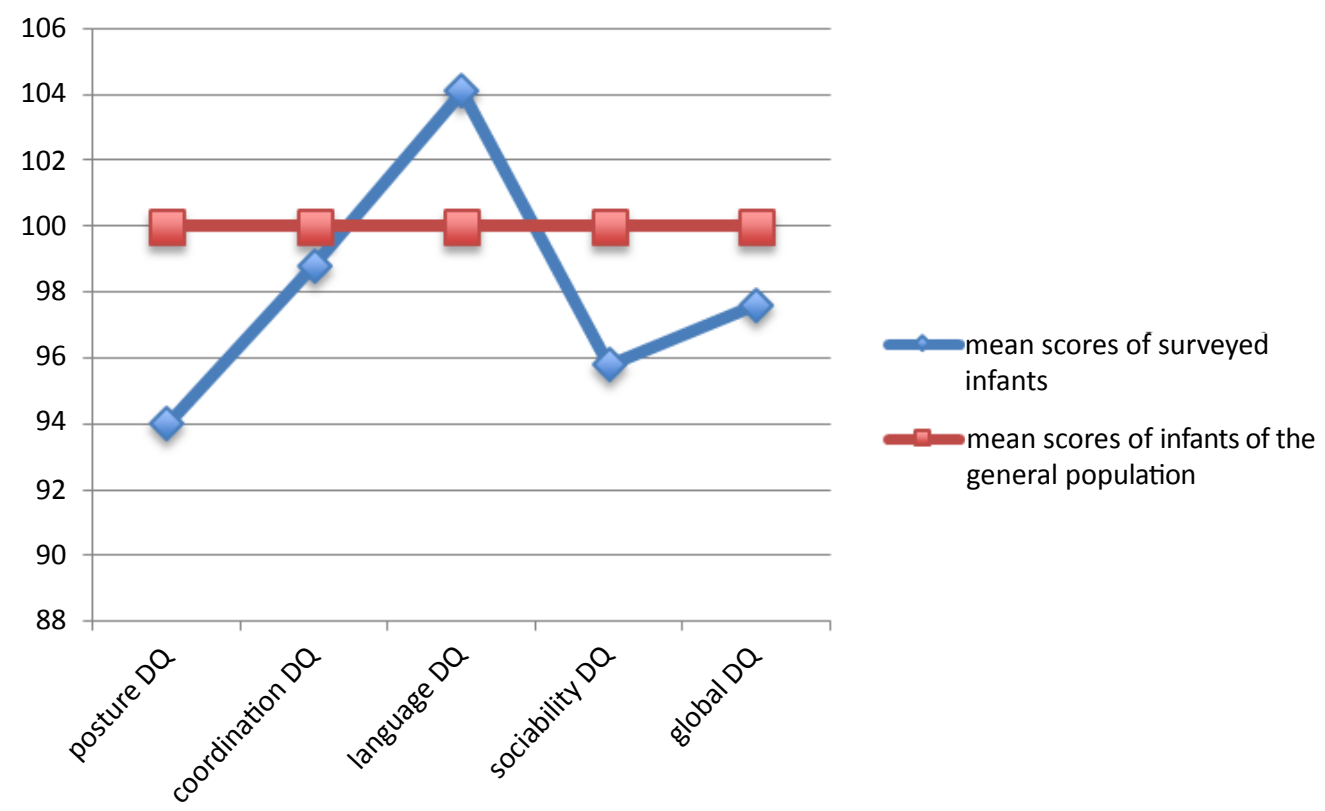

Figure 1: Mean subscores and global scores of the infants on the revised Brunet-Lézine scale. 
Citation: Le Treut L, Poinso F, Grandgeorge P, Dugnat M, Sparrow J, et al. (2017) Maternal Postpartum Depression and Infant Psychomotor Development: Descriptive Study of a Population Hospitalized in the Mother and Baby Unit. J Ment Disord Treat 3: 138. doi:10.4172/2471. 271X.1000138

Page 3 of 3

depression might have a particular impact on this type of interaction, which could explain the moderate drop in posture DQ scores.

These DQs were tested early, and the mothers also received psychiatric care that attenuated the severity of depressive symptoms and therefore any potential repercussions on child development. Furthermore, the infants who received early treatment in the mother and child unit had the advantage of having child care nurses with them. It is possible that this treatment, which focused on interactions and promoting interpersonal communication, boosted early socialization. We would therefore suggest that the treatment had a positive effect on infant development. Indeed, child care nursing could allow development of nonverbal communication and maternal positive feelings about parenting, which have been associated with resilience in children exposed to depressive symptoms postnatally (SavageMcGlynn, Redshaw, Heron, Stein, Quigley, Evans, Ramchandani and Gray, 2015). A comparative study with a control group of depressive mothers treated without specific children's care would allow us to confirm this hypothesis.

The main limitation was the population size. It appears necessary to continue recruiting for this study in order to confirm these results with a satisfactory statistical power.

The DQ subscores taken in the different areas should also be interpreted with caution, because they are based on a small number of items, and the behavior of very young children is labile and remains sometimes difficult to assess, even for seasoned psychologists.

Scores taken when the child is six months old could serve as a baseline; it might prove useful to retest over time in order to screen for a potential drop in scores, and correlate the scores with maternal symptom progression.

Despite the rather reassuring appearance of our results, we would like to draw attention to the advantages of a more objective metric than daily observations of a baby's behavior. This assessment is part of a systematic evaluation, useful in learning the baby's strengths and weaknesses; it can sometimes identify developmental warning signs.

This study is the first step before a larger study in which dyads will be prospectively followed with repeated development assessments on infants in parallel with assessment of maternal depression progression.

\section{References}

1. Poinso F, Gay MP, Glangeaud-Freudenthal NMC, Rufo M (2002) Care in a mother-baby psychiatric unit: analysis of separation at discharge. Arch Womens Ment Health 5: 49-58.

2. Cazas O, Glangeaud-Freudenthal NMC (2004) The History of Mother-Baby Units (MBUs) in France and Belgium and of the French version of the Marcé checklist. Arch Women's Ment Health 7: 53-58

3. Glangeaud-Freudenthal NMC, Sutter-Dallay AL, Thieulin AC, Dagens V Zimmermann MA, et al. (2013) Predictors of infant foster care in cases of maternal psychiatric disorders. Soc Psychiatry Psychiatr Epidemiol 48: 553-561.

4. Winnicott DW (2008) De la pédiatrie à la psychanalyse, Paris

5. Murray L, Arteche A, Fearon P, Halligan S, Croudace T, et al. (2010) The effects of maternal postnatal depression and child sex on academic performance at age 16 years: a developmental approach. J Child Psychol Psychiatry 51: 1150-1159.

6. Cornish AM, McMahon CA, Ungerer JA, Barnett B, Kowalenko N, et al. (2005) Postnatal depression and infant cognitive and motor development in the second postnatal year: The impact of depression chronicity and infant gender. Infant Behav Dev 28: 407-417.

7. Fraiberg L, Fraiberg S (1980) Clinical studies in infant mental health: the first year of life. New York, USA.

8. Brunet O, Lézine I, Josse D (1997) Brunet-Lézine révisé: échelle de développement psychomoteur de la première enfance: BLR. Editions et applications psychologiques.

9. Bayley N (1993) Bayley scales of infant development: manual. Psychological Corporation.

10. Wallon H (2015) Les origines du caractère chez l'enfant: les préludes du sentiment de personnalité.

11. Ajuriaguerra J de (1974) Manuel de psychiatrie de l'enfant (2éd) Paris: Masson.

12. Bullinger A (2004) Le développement sensori-moteur de l'enfant et ses avatars: Un parcours de recherche. Ramonville Saint-Agne: Erès. 